\title{
Adapted Wavelets for Pattern Detection
}

\author{
Hector Mesa ${ }^{1,2}$ \\ 1 University of La Habana, \\ Faculty of Mathematics and Computer Sciences, \\ 10400 La Habana, Cuba \\ hectormesa@matcom.uh.cu \\ 2 Paris-Sud XI University, 91400 Orsay, Paris, France \\ hector.mesa@math.u-psud.fr
}

\begin{abstract}
Wavelets are widely used in numerous applied fields involving for example signal analysis, image compression or function approximation. The idea of adapting wavelet to specific problems, it means to create and use problem and data dependent wavelets, has been developed for various purposes. In this paper, we are interested in to define, starting from a given pattern, an efficient design of FIR adapted wavelets based on the lifting scheme. We apply the constructed wavelet for pattern detection in the $1 \mathrm{D}$ case. To do so, we propose a three stages detection procedure which is finally illustrated by spike detection in EEG.
\end{abstract}

\section{Introduction}

The fields of application of wavelets grows because of their attractive properties for various purposes. The possibility to construct new wavelets with simplicity is one of the characteristics.

The construction of problem or data dependent wavelets have been undertaken by many authors: for instance, Zhang et al. 1 construct orthonormal wavelets bases which are best suited to represent a given signal, Lucas et al. [2] create orthogonal wavelets to improve the classification accuracy for certain given classes and Du et al. 3] use adapted wavelets for crackle detection.

The main contribution of the present paper is a modification of the Lifting Method to construct adapted wavelets introduced by Sweldens. Such wavelets are particulary well suited for pattern detection what we use for illustration. The modification overcomes the drawback of Sweldens approach concerning the too coarse approximation.

The paper is organized as follows. Section 2 gives some motivations to use pattern-matched wavelets as templates and proposes a three stages procedure to use them for pattern detection problems. In section 3 we propose a lifting based method to construct such pattern-adapted wavelets. Section 4 illustrates the procedure by considering the spike detection problem. 


\section{Pattern Detection in 1D with Adapted Wavelets}

For many applications, detection of known or a priori unknown patterns in signals are required. Those patterns can also be transformed (translated, scaled, etc), so it is usually needed to estimate the parameters of the transformation.

Here, we will suppose that we have to detect some translated and scaled versions of one given pattern. On the matter of this article, the pattern detection problem consists of, given a finitely supported pattern $f$ and a signal $S$, to find where the signal is similar to a scaled version of the pattern estimating both time-shift and scale factor.

\subsection{Why Adapted Wavelets for Pattern Detection}

Template matching or pattern matching is used for this purpose [4. Two methods are commonly used: signal substraction, where the norm of the difference is used as a measure of the dissimilarity, and the correlation, where the scalar product is considered as a measure of similarity. Both methods are equivalent when the template and the signal are normalized so they have zero average and norm 1. But a zero-average 1D finite supported function is close to a wavelet.

On the other hand, the wavelet transforms have efficient implementations and allow to decompose any signal in "frequency" bands. So, why not to use wavelets as templates, this is to use pattern-adapted wavelets.

A wavelet $\psi_{f}$ approximating any given pattern $f$ allows, by means of the wavelet transform, to estimate the correlation of any signal $S$, not only with the pattern itself, but with its scaled versions. The continuous wavelet transform (CWT) of a signal $S$ with the wavelet $\Psi$ at scale $a>0$ and time $b$ is defined as:

$$
W_{\Psi} S(a, b)=\left\langle S, \frac{1}{\sqrt{a}} \Psi\left(\frac{x-b}{a}\right)\right\rangle .
$$

The values $W_{\Psi}(a, b)$ are also called the wavelet coefficients of $S$ in $(a, b)$.

The discrete-like transforms - discrete wavelet transform (DWT) and translation invariant wavelet transform (SWT) - are fast algorithms to compute it for dyadic scales $\left(a_{j}=2^{j} a_{0}\right)$. It is calculated at scale-dependent shifts by the DWT $\left(b_{j, k}=b_{0}+j a_{k}\right)$ and at some fixed shifts $b_{j, k}=b_{0}+j$ by the SWT.

Also, for any fixed scale $a>0$, every local maxima of the similarity cause local maxima of the wavelet energy (squared wavelet coefficients), i.e. those pairs $(a, b)$ for which the wavelet energy is locally maximum as a function of $b$ are the only possible values for which the similarity between the signal and the corresponding scaled and translated versions of the pattern is locally maximum.

\subsection{A Three Stages Procedure}

So, we can search such wavelet energy maxima (called similarity alert or simply alert) and then to individually test if they are interesting with respect to the 
problem (true alert) or not (false alert). Then, to know if the alert is true or false it is needed to verify some rules.

The similarity is verified by using rules that are designed only from the pattern - we called them pattern-based rules -. The relevance with respect to the problem is checked by using problem dependent rules. Usually, neural networks are implemented to create such rules automatically from a training set of data 56 .

Resuming this idea we propose a three stages procedure:

1. Given the motif $f$ to detect, create the pattern adapted wavelet $\psi_{f}(x)$. Without loss of generality we will suppose that $\operatorname{supp}(f)=[0,1]$ (so that the scale $a$ represents the size of the corresponding pattern and $b$ the starting time), $\int_{0}^{1} f(x) d x=0$ and $\|f\|_{2}=1$.

2. Detect all the alerts on the signal. This is to search the local maxima of the signal wavelet energy for any $b$ and all possible, for the problem, durations $a>0$.

3. Detect and discard all the false alerts. For this some rules must be applied to decide if each alert is false or not.

\section{Pattern Adapted Wavelets}

Let us start this section by giving some basic ideas about wavelets. More can be found for example in [7].

Wavelets are sufficiently smooth functions with zero average. The wavelet transform is well localized both in time and in frequency, unlike the Fourier transform.

One important concept on the wavelet theory, is the Multiresolution Analysis (MRA). It is the base of discrete decomposition of signals in terms of translates and scaling of a single function and so of the computationally efficient algorithms to compute the DWT and SWT like the Mallat's "a trous" [7] which iteratively computes the wavelet transform for successive scale levels.

The MRA is a family $M=\left\{V_{j}\right\}_{j \in \mathbb{Z}}$ of nested closed subspaces of $L^{2}$ :

$$
\{0\} \subset \cdots \subset V_{1} \subset V_{0} \subset V_{-1} \subset \cdots \subset L^{2}
$$

named approximation spaces which satisfy the conditions stated for example in 8] p. 65. It can be defined another family of closed subspaces $\left\{W_{j}\right\}_{j \in \mathbb{Z}}$, called details spaces, such that $V_{j-1}=V_{j} \oplus W_{j}$.

The so called scaling function $\varphi$ and wavelet $\psi$ are so that their linear integer translates spans generate $V_{0}$ and $W_{0}$ respectively.

One of the most important consequences of the MRA definition is the existence of two filters $u$ and $v$ satisfying the two-scales relations:

$$
\left\{\begin{array}{l}
\frac{1}{\sqrt{2}} \varphi\left(\frac{x}{2}\right)=\sum_{k} u[k] \varphi(x-k) \\
\frac{1}{\sqrt{2}} \psi\left(\frac{x}{2}\right)=\sum_{k} v[k] \varphi(x-k)
\end{array} .\right.
$$


Those filters are known as the two-scales filters.

The fast algorithms take profit of this relation to compute the transform at dyadic scales. The invertibility of the fast algorithms are ensured by a new pairs of filters $\stackrel{\circ}{u} \stackrel{\circ}{v}$ satisfying the perfect reconstruction $(\mathrm{PR})$ property:

$$
\left\{\begin{array}{l}
u^{\vee} \star \dot{u}+v^{\vee} \star \dot{v}=2 \delta_{0} \\
\widetilde{u}^{\vee} \star \dot{u}+\widetilde{v}^{\vee} \star \stackrel{v}{v}=0
\end{array}\right.
$$

where $\delta_{k}$ is the unit response at time $k$ and where the convolution operator is denoted by $\star$ and the subsampling, upsampling, modulation and transpose of a filter $u$ are denoted by $[u]_{\downarrow_{2}},[u]_{\uparrow_{2}}, \widetilde{u}$ and $u^{\vee}$ respectively.

In the case of the existence of a biorthogonal MRA with scaling function $\dot{\varphi}$ and wavelet $\dot{\psi}$ in $L^{2}$, the associated filters satisfy (4). The second MRA, scaling and wavelet function, and filters will be called dual and denoted by appending a "०" (e.g. $\stackrel{\varphi}{)}$.

\subsection{Adapted Wavelet Construction}

There are many ways to construct wavelets. Some of those approaches are to create signal (or pattern) adapted wavelets e.g. [910].

Abry et al. 111213] propose to construct a time-space matched scaling function or wavelet by projecting the pattern onto the approximation spaces or detail spaces $\left(V_{0}, W_{0}\right.$ respectively) of some initially given MRA. Then, it is possible to compute the new functions as admissible (i.e. invertible) linear combinations of the original ones and to compute the associated filters.

Their approach allows to construct four new functions $\varphi, \psi, \stackrel{\circ}{\varphi}$ and $\dot{\psi}$ in $L^{2}$ satisfying the biorthogonality conditions and generating a pair of biorthogonal MRAs. As said above, the existence of such functions and MRAs, ensures the property (4). The main problem is that in general, when using this approach, the new associated filters may not exist or will have infinite impulse response (IIR), reducing the efficiency of the algorithms. So, truncating the filters is required but it may be the cause of large errors which will grow at each iteration compromising the convergence and the accuracy of the algorithms.

\subsection{Lifting Based Methods}

Principle. Another approach is the lifting method introduced by W. Sweldens 14 15. This method allows, starting from four filters $u, v, \stackrel{i}{u}, \stackrel{\circ}{v}$ satisfying the PR property (i.e. a perfect reconstruction filter bank PRFB), to construct a new PRFB. This method, for instance, allows to create second generation wavelets which are not necessarily translates and dilates of one fixed function [16]. Also, Sweldens shows that any discrete wavelet transform can be decomposed in (primal and dual) lifting steps, giving a new, more efficient, algorithm called fast lifting transform [17]. 
The idea of wavelet construction with a primal lifting step is, starting from a scaling function $\varphi$ and wavelet $\psi$ generating a MRA which are associated to $u$ and $v$, to make

$$
\psi_{l}(x)=\psi(x)+\sum_{i} l[i] \varphi(x-i)
$$

where $l$ is a finite filter with zero average.

The new PRFB would be $\left(u, v^{N}, \stackrel{\circ}{u}^{N}, \stackrel{\circ}{v}\right)$ with

$$
\begin{aligned}
& u^{N}=u-[l]_{\uparrow_{2}}^{\vee} \star v \\
& \stackrel{\circ}{v}^{N}=\stackrel{\circ}{v}+[l]_{\uparrow_{2}} \star \stackrel{\circ}{u} .
\end{aligned}
$$

The dual lifting step can be obtained by exchanging the primal functions and filters with the dual ones.

Let $f \in L^{2}$ be a normalized $\left(\|f\|_{2}=1\right)$ and compactly supported function with zero-average. To approximate $f$ by a wavelet function $\psi_{f}$ constructed with this method we have to project $f-\psi$ onto $V_{0}$ which gives us the lifting filter $l^{*}$ and so a pattern-matched wavelet

$$
\psi_{f}(x)=\psi(x)+l^{*} \star \varphi(x) .
$$

The approximation with this method can be too coarse as shown in Figure 11(b).

A Variant. To circumvent this drawback, we propose to use a dilated version of $f\left(f_{\rho}(x)=\frac{1}{\sqrt{\rho}} f(x / \rho)\right.$ where $\rho$ is the dilation coefficient) and a variant of the lifting step.

The dilation coefficient $\rho$ allows to take profit of the good approximation properties of the scaling functions [18] reducing $\left\|f-\mathbb{P}_{V_{0}} f\right\|$, where $\mathbb{P}_{V_{0}} f$ means the projection of $f$ onto $V_{0}$, but $\psi$ is still seen reducing the accuracy of the approximation (see Figure 1(c)).

We propose a variant to the lifting step which reduces the influence of $\psi$ in the constructed wavelet $\psi_{f}$. Let $l$ be a finite filter with $\sum_{k} l[k]=0$, a real number $c$ such that $|c|>c_{\text {min }}$ for some $c_{\text {min }}>0$ and an integer $k$. The new primal wavelet will be

$$
\psi_{l}(x)=c \psi(x-k)+\sum_{i} l[i] \varphi(x-i)
$$

and the associated filters $\left(u, v^{N}, \stackrel{\circ}{u}^{N}, \stackrel{\circ}{v}^{N}\right)$ where

$$
\begin{aligned}
& v^{N}=c \cdot v \star \delta_{2 k}+[l]_{\uparrow_{2}} \star u \\
& \dot{u}^{N}=u-\frac{1}{c} \delta_{2 k} \star[l]_{\uparrow_{2}}^{\vee} \star v \\
& \dot{\circ}^{N}=\frac{1}{c} \delta_{2 k} \star \stackrel{\circ}{ } .
\end{aligned}
$$


The new four filters also satisfy (4).

The use of a small enough $c$ and a convenient $k$ reduce the influence of $\psi$. The value of $c_{\min }$ must be chosen so that the dual filters $\dot{u}$ and $\dot{v}$ are not too large.

If $W_{0} \perp V_{0}$ and $\mathbb{P}_{W_{0}} f \not \equiv 0$, the optimal values of $c$ and $k$ can be obtained from the wavelet decomposition of $\mathbb{P}_{V_{-1}} f$ :

As

$$
\mathbb{P}_{V_{-1}} f=\mathbb{P}_{V_{0}} f+\mathbb{P}_{W_{0}} f,
$$

then taking $c^{*}, k^{*}$ such that

$$
k^{*}=\arg \max _{k}\left|W_{\psi}(0, k)\right|,
$$

and

$$
c^{*}=\operatorname{sign}\left(W_{\psi}\left(0, k^{*}\right)\right) \max \left(\left|W_{\psi}\left(0, k^{*}\right)\right|, c_{\text {min }}\right) .
$$

where $\operatorname{sign}(\cdot)$ denotes the sign function.

If $\mathbb{P}_{W_{0}} f \equiv 0$ then $k^{*}$ is free and $c^{*}=c_{\min }$.

When $W_{0} \not \perp V_{0}$ then an optimization problem has to be solved:

$$
\min _{\sum_{k} l[k]=0,|c|>c_{\text {min }}}\left(\|f-c \psi(x-k)-l \star \varphi(x)\|^{2}\right) .
$$

Resuming, projecting $f$ onto $V_{0}$ gives $l^{*}$ and for the convenient values of $c^{*}$ and $k^{*}$ we get the pattern-matched wavelet.

$$
\psi_{f}(x)=c^{*} \psi\left(x-k^{*}\right)+l^{*} \star \varphi(x) .
$$

An example of a pattern-adapted wavelet computed using this method can be seen in Figure 1(d).

Illustration. Let us consider $f(x)=\sqrt{3}(.5-2|x-.5|) \mathbb{1}_{[0,1]}$ (see Figure 1(a)) as motif. Let us use Db5's MRA, this is the MRA generated by the scaling function and wavelet with 5 vanishing moments obtained by Daubechies [19], for which $V_{0} \perp W_{0}$.

Figure 1(b) shows the wavelet constructed using a classical lifting step. Due to the small support of $f([0,1])$, the new wavelet is not well adapted to the pattern. By approximating $f_{\rho}$, a dilated version of $f$, with $\rho=16$ we get a much better solution but the influence of the original wavelet is evident.

Now, using our lifting's variant step for $\rho=16$ (see 1(d)), we get better results. Notice that the original wavelet has almost disappeared.

Properties. This construction method is stable for small variations in the pattern:

Let $g \in L^{2}$ be a function such that $\|g\|_{2}=1$. Let $f^{\varepsilon}(x)=f(x)+\varepsilon g(x)$ for any $\varepsilon \in \mathbb{R}$. We have that

$$
\left\|\psi_{f}-\psi_{f}^{\varepsilon}\right\|^{2} \leq \varepsilon^{2} \cdot{ }^{T} \sigma_{g}(H)^{-1} \sigma_{g},
$$




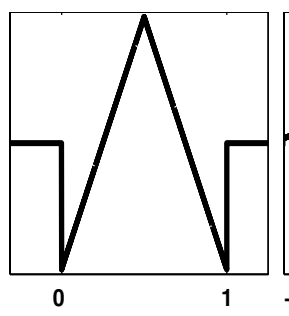

(a)

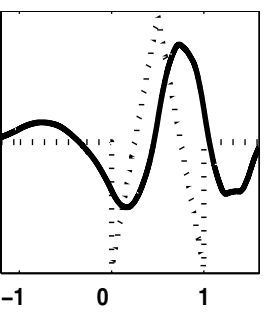

(b)

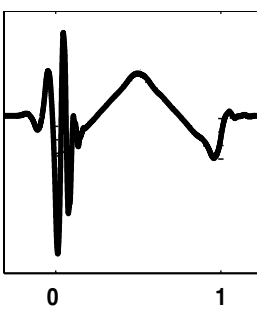

(c)

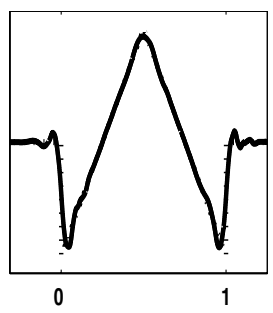

(d)

Fig. 1. Example of pattern adapted wavelets construction using lifting-based methods starting from Db5's MRA. (a) the pattern $f$, (b) the wavelet obtained by an original lifting step, (c) the wavelet obtained by using the original lifting step but $f$ was dilated with $\rho=16$ and (d) wavelet created by using our variant and $\rho=16$.

where $\sigma_{g}[i]=\langle g, \varphi(x-k)\rangle, H[i, j]=\gamma[i-j]$ and $\gamma[i]=\langle\varphi, \varphi(x-i)\rangle$ is the sampled autocorrelation function.

Continuity and differentiability properties of the constructed wavelet are ensured by the original scaling function's properties and it is possible to obtain an arbitrary number of vanishing moments by adding some linear constraints, besides the l's null sum restriction, while keeping the stability of the method. Unlike the projection methods, the dual functions may not have finite energy but the PR property hold so the analysis-synthesis algorithms work.

The good behavior of such adapted wavelets for pattern detection to find possible points (in the time-scale plane) of locally maximum similarity is shown in Figure 2(a) where it is represented the CWT of a fragment of an EEG with a wavelet adapted to a simple model of a spike-wave complex (a spike followed by a slower wave) (Figure 3 (a)) which we want to detect.

Three maxima (one with positive coefficient in the center and two negatives on both sides) are present for each complex location but a further analysis shows that the true location is represented by center ones (all for scales between $a=.2$ and .3 ).

Figures 3(b) and (c) show the scaled and shifted versions of the adapted wavelet for two consecutive local maxima ( $b=.0985$ and $b=1.125$ respectively). They are also multiplied by the estimated amplitude. See that in the first case, although the similarity is high, it is lower than this of the second case (fig [3(c)) where there is an almost perfect match. Those false maxima must be eliminated by selection rules like to check the possible scales or duration (in this example, the complexes have a duration around .22s) or by direct testing if there are only a few of local maxima to check.

\section{Real World Example: Spike Detection in EEG}

Electroencephalogram (EEG) is an important clinical tool for diagnosing, monitoring and managing neurological disorders related to epilepsy. Spikes correspond to tiny epileptic discharges which last for a fraction of a second. Beyond 
(a)
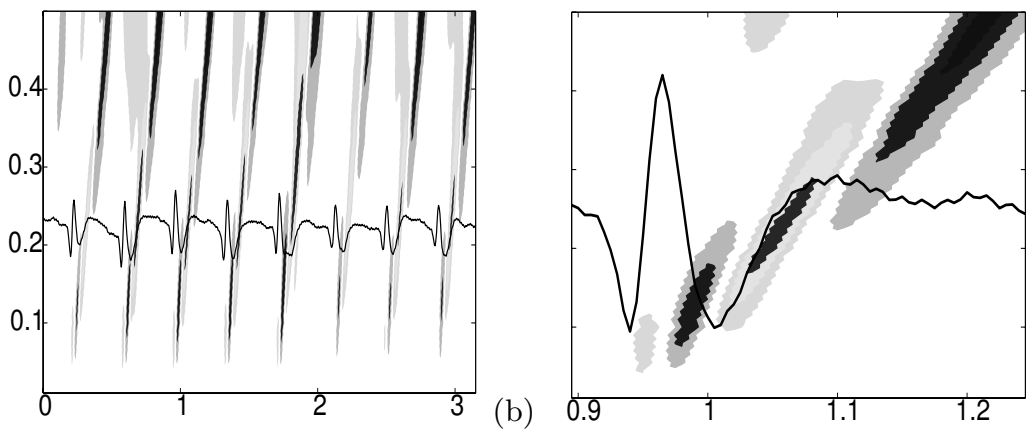

Fig. 2. (a)An EEG fragment and its CWT with the spike-wave complex adapted wavelet. (b)A zoom between .9 and 1.2s shows the existence of three local maxima of the wavelet energy around a spike-wave complex.

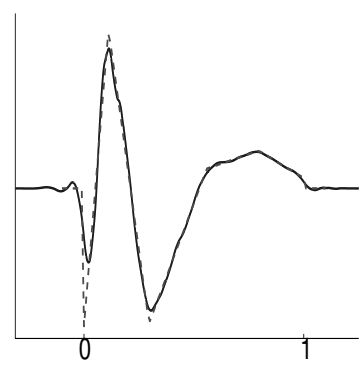

(a)

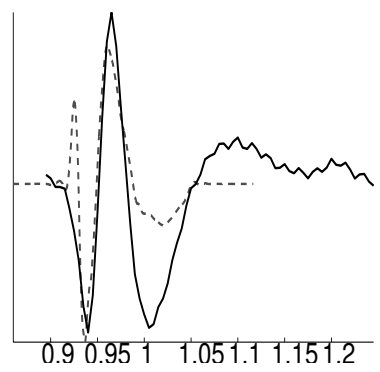

(b)

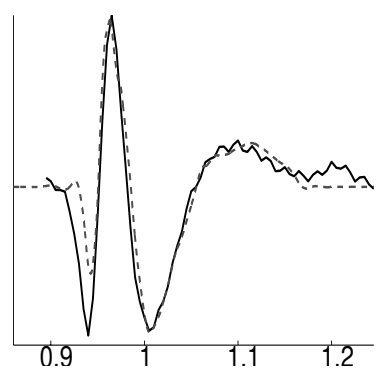

(c)

Fig. 3. (a)Spike-wave complex model (dashed line) and the adapted wavelet(solid line). The EEG signal with scaled $(a)$ and shifted $(b)$ adapted wavelet: (b) for $b=.985 \mathrm{~s}$ and $a=.125$, (c) for $b=1.05 s$ and $a=.225$.

of the diagnosis of epilepsy, automatic spike detection helps to make quantitative descriptions of spike density, topology and morphology what could help to determine patient syndrome and surgical outcome [20].

Spike detection is tedious and requires skill to do it well. The noise and the artifacts make this task difficult [21. That is why there are so many people working on automatic spike detection. We use this problem to illustrate the behavior of this procedure as an example of the use of the pattern-matched wavelets. Let us first describe the problem.

\subsection{The Problem}

The epileptic spikes on the EEG was loosely defined by Gloor in 22]. He gives three characteristics regarding their form (a restricted triangular transient), its duration (having a duration of $\leq 200 \mathrm{~ms}$ ) and the electric field (as defined by involvement 
of a second adjacent electrode). He says also that the spikes must be clearly distinguishable from background activity and having an amplitude of, at least, twice of the preceding 5 s of background activity in any channel of the EEG.

Many works roughly follow this definition and emphasize the local context, morphology and the field of the spike. As local context it is understood the local characteristics of the signal compared with the background activity. The term "background activity" is used to describe the context in which the spikes occurs and it is typically used to normalize the spikes parameters to account for varying electrical output from different patients and determine whether the spike is more than a random variation of the underlying rhythmic activity [20].

As morphology it is understood every attribute used to describe the spike and its background. Relative height, relative sharpness at the apex and total duration are some examples of attributes.

To detect the presence of field it is needed to analyze adjacent electrode signals. Overlapping spikes on different channels are used to create a spike-event [23] so it is used as a rule to discard false alerts.

\subsection{The Detection Procedure}

First we have to choose the pattern. From the first characteristic given by Gloor, we take a triangular function (Figure1(a)) as a very simple pattern. We will use Db5's as initial MRA, whose wavelet is two times continuously differentiable, and $\rho=16$. The resulting wavelet is shown in Figure 1(d).

The alert detection process for a signal $S$ consists in the detection of local maxima in $b$ of the wavelet energy $W_{\psi_{f}}^{2} S(a, b)$ for every scale $a>0$. It can be done for dyadic scales, with the DWT or SWT, or for more regularly spaced scales, by using the CWT.

This will give us the times when the spike-alerts occur. To decide the optimum scale $a^{*}$ (i.e. the duration) for any alert time $b^{*}$, it is needed to compare between the adjacent scales for the times $b$ such that the wavelet supports intercept. We will take $\frac{1}{a^{3}} W_{\psi_{f}}^{2}(a, b)$ as an estimation of spikes slopes and will keep those alerts where it is locally maximum as a two-variables function.

\subsection{Some Selection Rules}

Pattern-Based Rules. Those rules consist of the threshold of upper bounds of the approximated similarity between the pattern and the signal's fragment. Two possible upper bounds of the similarity are

$$
\operatorname{corr}\left(a^{*}, b^{*}\right) \leq\left(1+\frac{\left|W_{\psi_{f}} S(a, b)-\Gamma^{*} \cdot W_{\psi_{f}} S\left(a^{*}, b^{*}\right)\right|}{\left|W_{\psi_{f}} S\left(a^{*}, b^{*}\right)\right| \sqrt{1-\Gamma^{* 2}}}\right)^{-1}
$$

and

$$
\operatorname{corr}\left(a^{*}, b^{*}\right) \leq\left(1+\left|\frac{a^{* 2} \partial_{b, b}^{2} W_{\psi_{f}} S\left(a^{*}, b^{*}\right)+W_{\psi_{f}} S\left(a^{*}, b^{*}\right)\left\|\psi_{f}^{\prime}\right\|^{2}}{\left|W_{\psi_{f}} S\left(a^{*}, b^{*}\right)\right| \sqrt{\left\|\psi_{f}^{\prime \prime}\right\|^{2}-\left\|\psi_{f}^{\prime}\right\|^{2}}}\right|\right)^{-1}
$$


where $\Gamma^{*}=\left\langle\psi_{f}(x), \psi_{f}\left(\frac{x-\left(b-b^{*}\right) / a^{*}}{a / a^{*}}\right)\right\rangle$ is the correlation function of $\psi_{f}$ and $\partial_{b, b}$ means the second derivative twice in $b$. Those bounds can be easily evaluated from some selected values of the pair $\left(a / a^{*}, \frac{b-b^{*}}{a^{*}}\right)$.

Problem-Dependent Rules. Now, as every pattern-similar event will satisfy those conditions, it is necessary to add some problem-dependent selection rules.

As $\operatorname{supp}(f)=[0,1]$, from the characteristics given by Gloor, we have that $a \leq .2$. To discard some possible discontinuities we will keep only all scales $a>.01$ for EEG sampled at $200 \mathrm{~Hz}$. Hence the first rule is that the possible scales are in the range $[.01, .2]$, so, adding one scale up and one down of the range in the analysis, we can discard those events whose duration is not in the range: if the estimated slopes for any of those out-of-range scales is larger than those corresponding to the scales in the range, then this alert is discarded.

Other rules are taken from the facts that the spikes must be distinguishable, with an amplitude of at least twice that of the background, must be more than a random event and must cause a field. The first three rules depend on each channel independently and the last include other adjacent channels in the analysis. As a measure of the average background amplitude we can use the average of the locally maximum wavelet energy for $5 s$ of signal before the alert-time. The rule consists in normalizing the wavelet energies by the average background amplitudes and comparing it with a threshold $\tau_{\sigma}>=4$.

To keep only those events that are not caused by random effects, we take those whose instant wavelet energy is larger than a multiple of the standard deviation $\sigma$ of the background amplitudes plus its mean $\mu$.

\subsection{Spike Detection Results}

Here we show some results of the procedure with the described rules. Figures 4 and 5 show two different EEG with the detected spikes. Each figure represents

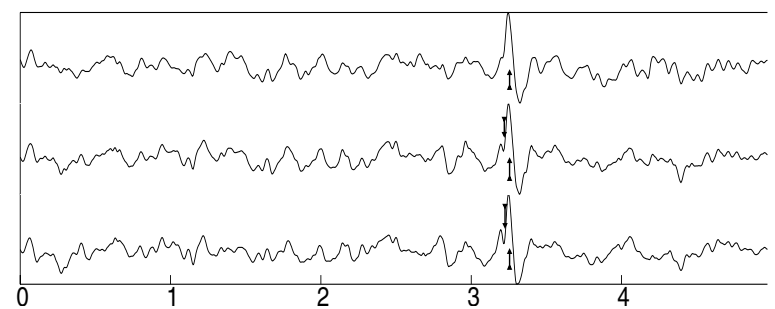

(a)

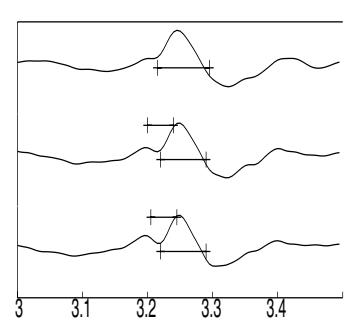

(b)

Fig. 4. A first example of spike detection results performed on three different channels independently of each other. (a)The EEG and the detected locations which are signaled by an arrows. (b) Zoom of (a) between 3 and 3.5s. The interval duration of the detected events are marked as line segments limited by two crosses ('+'). 


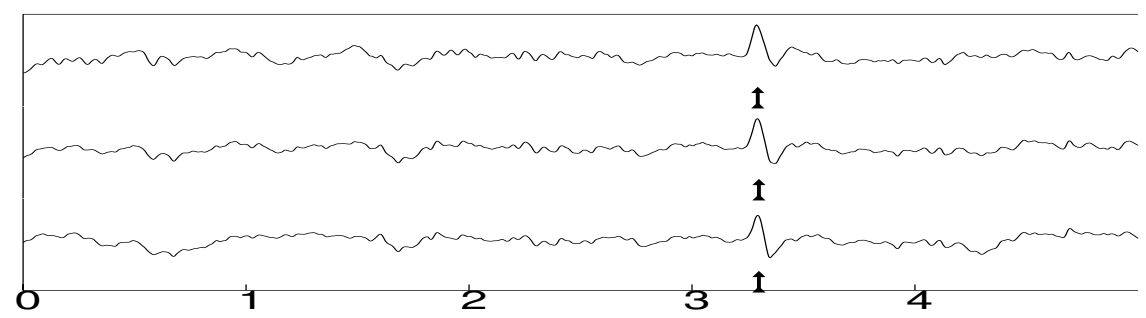

Fig. 5. A second example of spike detection results performed on three different channels independently of each other. The detected locations are signaled by an arrow.

three adjacent channels of the same EEG. Notice that there exists spike events almost simultaneously in various channels, i.e. there exists a field for those spikes.

Figure 4(b) represents a zoom of Figure 4(a) between 3 and 3.5s. The interval of duration of the detected events are signaled as line segments limited by two crosses ('+'). Notice the inverted spike-event overlapping the other (larger) spike in the second and third channels represented in Figure 4 by the up-down arrows.

\section{Conclusions}

To end, let us give some concluding remarks for future work. First, this paper shows how the lifting methods can be used to construct pattern-adapted wavelets. Unlike Abry et al. approach, they always give FIR filters but the dual functions may have infinite energy. An additional analysis must be done when $L^{2}$ dual functions are required. Second, as many of the pattern detection problems are for images, the generalization of this method to the $2 \mathrm{D}$ case will be done but such pattern-adapted wavelet construction method is more difficult. Since efficient 2D wavelets are associated to MRAs obtained by tensor products of the 1D wavelet filters and many possible patterns cannot be well approximated by such wavelets. Finally, another problem to solve is that, up to now, we start with a pattern given a priori and separately but for many applications such motifs are noisy or have to be taken from some experimental signals [24].

\section{References}

1. Zhang, J.K., Davidson, T.N., Wong, K.M.: Efficient design of orthonormal wavelet bases for signal representation. IEEE Transactions on Signal Processing 52 (2004) 1983-1996

2. Lucas, M.F., Hitti, E., Doncarli, C.: Optimisation d'ondelettes pour la classification. 18ième colloque GRETSI sur le traitement du Signal et des Images, Toulouse, France (2001)

3. Du, M., Chan, F.H.Y., Lam, F.K., Sun, J.: Crackle detection and classification based on matched wavelet analysis. In: Engineering in Medicine and Biology Society. Volume 4., Chicago, IL, USA, 19th Annual International Conference of the IEEE (1997) 1638-1641 
4. Brunelli, R., Poggio, T.: Template matching: Matched spatial filters and beyond. Pattern Recognition 30 (1997) 751-768

5. Ozdamar, O., Kalayci, T.: Detection of spikes with artificial neural networks using raw EEG. Computers and Biomedical Research 31 (1998) 122-142

6. Kalayci, T., Ozdamar, O.: Wavelet processing for automated neural network detection of EEG spikes. IEEE Med. Engng. Biol. 14 (1995) 160-166

7. Mallat, S.: A wavelet tour of signal processing. Academic Press (1998)

8. Misiti, M., Misiti, Y., Oppenheim, G., Poggi, J.M.: Les ondelettes et leurs applications. Hermes (2003)

9. Chapa, J.O., Rao, R.M.: Algorithms for designing wavelets to match a specified signal. IEEE Transactions on Signal Processing 48 (2000) 3395-3406

10. Fung, C.C., Shi, P.: Design of compactly supported wavelet to match singularities in medical images. Applications of Digital Image Processing XXV (2002) 358-369

11. Abry, P.: Ondelettes et turbulences. Diderot Editeur, Paris (1997)

12. Aldroubi, A., Unser, M.: Families of multiresolution and wavelet spaces with optimal properties. Numer. Funct. Anal. and Optimiz. 14 (1993) 417-446

13. Abry, P., Aldroubi, A.: Designing multiresolution analysis-type wavelets and their fast alghorithm. The journal of Fourier Analysis and Applications. 2 (1995) 135159

14. Sweldens, W.: The lifting scheme: A new philosophy in biorthogonal wavelet constructions. In Laine, A., Unser, M., eds.: Wavelet Applications in Signal and Image Processing III, Proc. SPIE 2569 (1995) 68-79

15. Sweldens, W.: The lifting scheme: A custom-design construction of biorthogonal wavelets. Appl. Comput. Harmon. Anal. 3 (1996) 186-200

16. Sweldens, W.: The lifting scheme: A construction of second generation wavelets. SIAM J. Math. Anal. 29 (1997) 511-546

17. Sweldens, W., Daubechies, I.: Factoring wavelets transform into lifting steps. J. Fourier Anal. Appl. 4 (1998) 247-269

18. Strang, G., Nguyen, T.: Wavelets and Filters Banks. Wellesley-Cambridge Press (1996)

19. Daubechies, I.: Ten Lectures on Wavelets. 3rd. edn. CBMS-NSF (1994)

20. Wilson, S.B., Emerson, R.: Spike detection: a review and comparison of algorithms. Clinical Neurophysiology 113 (2002) 1873-1881

21. Varsta, M., Heikkonen, J., Millan, J.R.: Epileptic activity detection in EEG with neural networks. Research Reports B3, Laboratory of Computational Engineering, Helsinki University of Technology (1998)

22. Gloor, P.: Contributions of electroencephalography and electrocorticography in the neurosurgical treatment of the epilepsies. Adv. Neurol. 8 (1975) 59-105

23. Wilson, S.B., Turner, C.A., Emerson, R.G., Scheuer, M.L.: Spike detection. Clinical Neurophysiology 110 (1999) 404-411

24. Scott, C., Nowak, R.: TEMPLAR: A wavelet based framework for pattern learning and analysis. IEEE Transactions on Signal Processing 52 (2004) 2264-2274 